\title{
Capillary Stop Valve Actuation by Thermo-pneumatic- pressure for Lab-On-Chip Systems
}

\author{
Ujjal Barman ${ }^{a, b *}$, Liesbet Lagae ${ }^{a, b}$, Benjamin Jones ${ }^{b}$ \\ a Department of Physics and Astronomy, KU Leuven, Celestijnenlaan 200d 3001 Heverlee Belgium \\ b Life Science Technologies Department, Imec, Kapeeldreef 75, 3001 Heverlee Belgium \\ *Corresponding Author: e-mail: barman.ujjal@gmail.com, phone: +91 9101900124
}

\begin{abstract}
A novel method is presented for triggering a robust capillary stop valve fabricated in silicon using the thermal expansion of trapped air bubble ( with a footprint of just $300 \mu \mathrm{m} \mathrm{x}$ $320 \mu \mathrm{m}$ ) as the actuation element. A heating element on the backside of a bubble trap chamber is utilized for thermal expansion of the air bubble. A voltage pulse of around $6 \mathrm{~V}$, the capillary barrier, around $1400 \mathrm{~Pa}$ was easily breached. A non-dimensionalized model has been developed using equivalent circuit model to describe the complex thermal/hydraulic behavior of the system. The trapped gas bubble temperature is input as a function of time in the model. A thermal finite element-based simulation is conducted to determine the gas temperature from the experimentally measured heater temperature. The model results are validated against experiments to aid in characterizing the dynamics of the problem.
\end{abstract}

Keywords. Thermo-pneumatic, capillary stop valve, hydraulic-capacitance, microheater, air bubble.

\section{Introduction}

The relation of microvalves to a lab-on-a-chip (LOC) system is that of the heart to a circulatory system of an animal. A LOC performs its job through operations like separation, mixing, heating, biochemical reactions, etc. on biological samples using chemical reagents (Temiz et al. 2015). Microvalves can control both the quantity and timing of fluid transport within a biochip (Oh and Chong 2006). The greatest challenge, however, is the integration of these essential components into a larger microfluidic platform. Many microvalve designs have been proposed and their performance demonstrated successfully, often as standalone devices (Grover et al., Hosokawa and Maeda 2000); however, the microfluidic literature do not have many instances where these designs can be implemented or integrated to a LOC system. There are some exceptions though like the microfluidic large-scale integration (Melin and Quake 2007) where they demonstrated biological protocol automation using thousands of mechanical valves and control components using pneumatic control on a single platform constructed with multilayers of PDMS on silicon substrate. The main disadvantage of this system is the control aspect of the microvalve. For standalone platforms, any control peripherals (Shaegh et al. 2015, Pilarski et al. 2005) such as pneumatic pressure source are not desirable. Another notable work reported by Juncker et al. 2002 where pumping, valving, and synchronization of processes are performed by a capillary powered autonomous system. This system requires a large footprint of the chip to accommodate timing channels for capillary trigger valves which makes the 
system expensive. Also, the surface properties vary with time altering capillary flow in the channels which shortens the self-life of the chip.

In short, an ideal microvalve should be easy to fabricate and integrate with a larger platform with on-chip flow control. It should have low power, current and voltage requirements as well. These motivated us to adopt a passive-active approach for microfluidic control. Capillary stop valves are an excellent choice since they use surface forces and microstructure design for restricting fluid flow. Capillary flow is the phenomenon by which liquid molecules in the bulk are brought to the gas-liquid interface thereby creating new surface. This is powered by surface energies provided by the microchannel surface. Fluid flow would cease if the energy provided by the surface is not enough to create new surface. This can be achieved by changing hydrophilicity of the surface or an abrupt change in geometry of the microchannel. Both these modifications (surface and geometric respectively) are known as capillary stop valves (Irimia 2008). A careful design of fabrication flow process of a larger system can incorporate such stop valves easily and simultaneously with other components. This solves the integration problem as well.

For resumption of flow, a suitable actuation mechanism of the stop valve is required which would breach the capillary barrier of the structure (Irimia 2008). Several novel methods have been successfully employed to do this like pressure from bubble generated by electrolysis reported by Man et al. 1998, by lowering the surface tension opto-thermally by using a laser directed at the valve meniscus demonstrated by Eriksen et al. 2017 or by means of centrifugal force as described in Cho et al. 2007 etc.

We adopt thermo-pneumatic pressure in this paper for the actuation of capillary stop valve. Some earlier works have already been done on thermo-pneumatic principle. Abi-Samra et al. 2011 has demonstrated thermo-pneumatic pumping for centrifugal microfluidic platform, van der Wijngaart W et al. 2007 demonstrates control of fluid flow using gas-liquid interface as a switch inside a microchannel whereas, Handique et al. 2000 shows droplet dispensing using this mechanism. Bohm S 2006 is the underlying idea of our work which claimed that a capillary stop valve can be actuated using this principle.

We have successfully demonstrated the working of a capillary stop valve and its actuation mechanism within the framework of the fabrication process based on our earlier work on micro-PCR chip where, we successfully performed DNA amplification using integrated microheaters (Barman et al. 2018). Having fixed the fabrication process, we zeroed in on electro-thermo-pneumatic expansion of trapped air bubble as the pressure source for breaching the capillary barrier of the stop valve. The choice was obvious because we already had the heating element in the chip.

This device can be operated at low voltages ( 3-6V) and temperatures $(\sim 308-340 \mathrm{~K})$, is compact $(300 \mu \mathrm{m} \times 320 \mu \mathrm{m}$ footprint) and has fast response time due to electrical based actuation. The stop valve meniscus has very high retention time i.e. no leakage issues. The mechanism of the device is discussed in section 2. To have a good insight into the working of the system an analytical model based on a reduced order model is proposed detailed in section 3. In the subsection 3.3, a fully coupled electrothermal finite element simulations to aid the model as heat input is performed on a full chip size computational domain. Fabricated device and its various components are depicted in section 4. In section 5, the experimental set up is elaborated with a representative figure. The results follow in section 6; we discuss the actuation 
of the valve with de-ionized water as working fluid and finally conclude with a comparison between the results obtained from experimental and analytical model.

\section{Device design and working mechanism}

The device schematics along with its working principle is depicted in Fig. 1. The device (see Fig. 1a has an inlet cum fluid reservoir connected to a microchannel (140 $\mu \mathrm{m}$ width) which narrows down to $5 \mu \mathrm{m}$ width to form a hydraulic resistance upstream of the valve. The microchannel then bifurcates in two directions: one along the plane of the microchannel, which expands abruptly into a closed chamber; the other, which has a circular cross section, takes a route perpendicular to the plane of microchannel and terminates at a capillary stop valve structure, with radius $\mathrm{a}=50 \mu \mathrm{m}$. On the opposite side of the stop valve is an open, uncovered microchannel. On top of the closed chamber, an aluminum microheater is positioned on the open silicon surface.

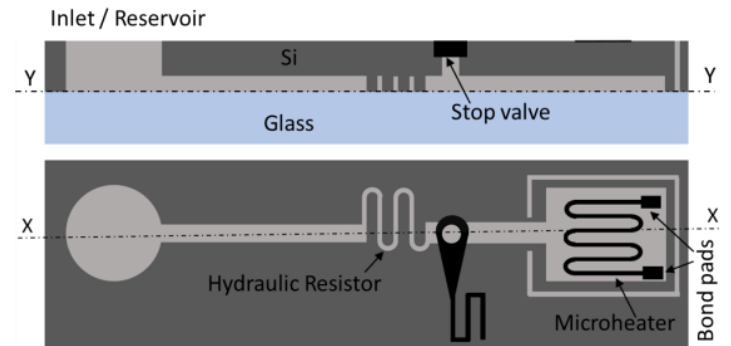

(a)

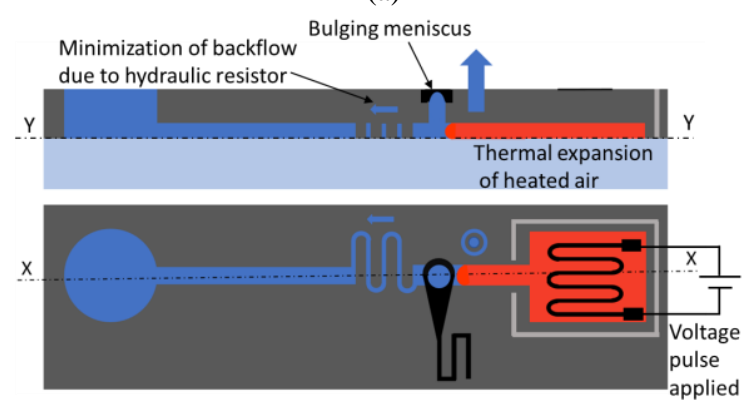

(c)

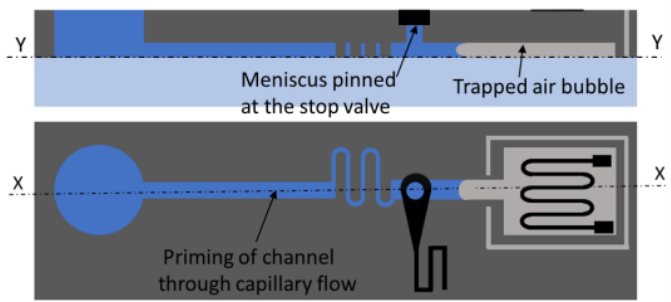

(b)

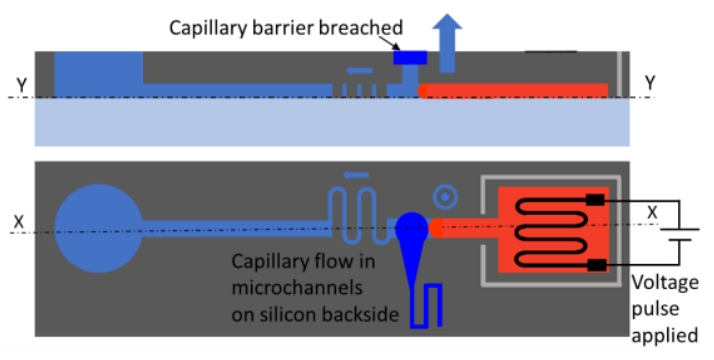

(d)

Fig. 1 Schematic of the electro-pneumatic valve test device and its operation: (a) cross-sections X-X and Y-Y of device, (b) device primed with fluid and waiting to be triggered, (c) voltage is provided to heat and expand the trapped air bubble, and (d) valve is actuated by breaching the capillary barrier; capillary flow commences in the microchannel on the silicon side.

As the device is primed (Fig. 1b) by capillary flow, fluid flows through the hydraulic resistor, past the bifurcation, and halts at the capillary stop valve while trapping an air bubble in the closed chamber. When an electric potential pulse is supplied to the joule heater, the trapped air bubble undergoes thermal expansion and displaces the liquid. The bulk of the liquid flow is directed towards the microvalve because of the high impedance towards the reservoir presented by the hydraulic resistor. The pinned meniscus is deformed (Fig. 1c) due to incoming fluid and when the liquid-gas interface at the contact line attains an angle equal to the contact angle with the top side of the silicon surface, the capillary barrier is breached and the meniscus advances radially (ideal condition) as a combination of pressure and capillary driven flow open surface of the tapered microchannel. The microvalve is surrounded by a taper shaped structure so that 
capillary force is enhanced as the meniscus proceeds towards the open surface microchannel. Eventually, the meniscus comes in contact with the side walls of the tapered microchannel and capillary pumping action of the microchannel actuates the valve. After the actuation, the pressure from the expansion gas is no more needed and the flow can be purely capillary driven (Fig. 1d).

\section{Analytical model}

The combined action of electrical conduction, heat transfer and fluid dynamics makes the system characteristics non-trivial to analyze. Here, we propose a simplified model of the system to capture the essence of the problem. We propose a thermo-hydraulic model based on the analogy between electrical and fluidic elements (Oh et al. 2012) due to the similarities in the transport phenomena of fluid and electrical charge. Such an equivalent network is based on steady state, fully developed, inertia less flow characteristic of a microfluidic system operating below its resonance frequency (Oh et al. 2012). The electrical potential gradient across an ohmic resistor mimics the pressure gradient across a hydraulic/fluidic resistor. Any accumulation of mass of fluid due to deformable elements in the fluidic circuit is replaced by an electrical capacitor (see Fig. 2a). Using these guidelines, we reduce the fluidic network to an equivalent electrical circuit. A pressure jump (Laplace pressure) due to gas-liquid interface can be represented by a voltage source. This greatly reduces the effort and computational cost of simulation at the system level.

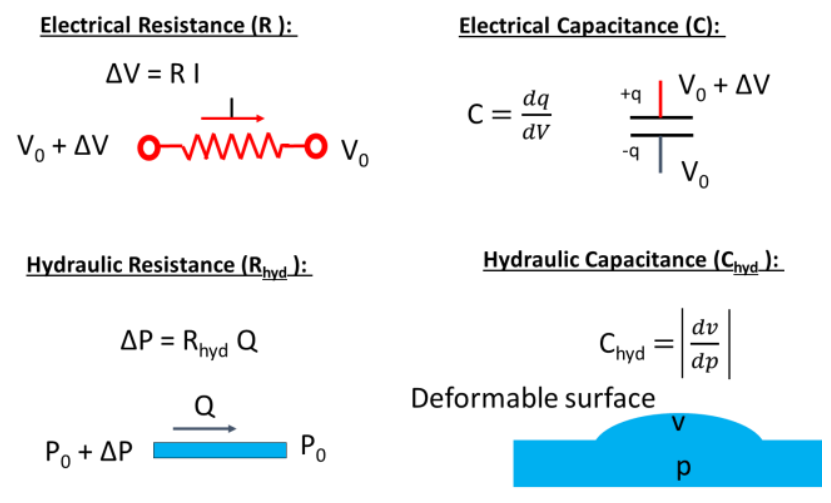

(a)

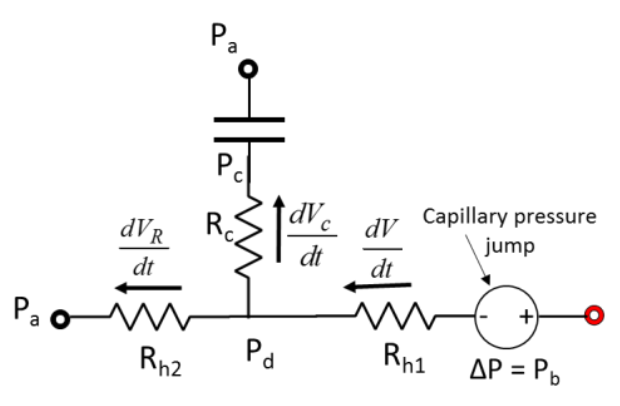

(b)

Fig. 2 (a) Equivalent electrical circuit elements for fluidic elements and (b) equivalent circuit model for the thermopneumatic valve based on the analogy.

Fig. $2 b$ shows the equivalent circuit model. Note that the initial pressure of the trapped air bubble is $\mathrm{P}(\mathrm{t}=0)=\mathrm{P}_{0}=\mathrm{P}_{\mathrm{a}}+\mathrm{P}_{\mathrm{b}}$, where $\mathrm{P}_{\mathrm{a}}$ is the atmospheric pressure and $\mathrm{P}_{\mathrm{b}}=2 \gamma\left(1 / \mathrm{h}_{1}+1 / \mathrm{w}_{1}\right)$ $\cos \theta$ is the capillary pressure jump across the meniscus trapping the air bubble with initial volume $\mathrm{V}_{0}$ and contact angle $\theta$. Here, $\gamma$ is surface tension of fluid, $\mathrm{h}_{1}$ is height and $\mathrm{w}_{1}$ is width of the microchannel designated with a resistance of $R_{h 1}$ in Fig. $2 b$.

\subsection{Fluid Network Analysis}

The liquid in the microchannel linking the gas chamber to microvalve is displaced at the same rate, $\mathrm{dV} / \mathrm{dt}$, as the rate of expansion of gas in the chamber. Furthermore, by mass conservation, $\mathrm{dV} / \mathrm{dt}$ equals the rate at which fluid is accumulated by the deforming capillary surface of the stop valve, $\mathrm{dV}_{\mathrm{c}} / \mathrm{dt}$, plus the flow rate through the hydraulic resistor, $\mathrm{R}_{\mathrm{h} 1}$, in the direction of the reservoir, $\mathrm{dV}_{\mathrm{R}} / \mathrm{dt}$ : 


$$
\frac{d V}{d t}=\frac{d V_{c}}{d t}+\frac{d V_{R}}{d t}
$$

By electrical analogy, from Fig. 2b, this can be written as:

$$
\frac{d V_{s}}{d t_{s}}=\frac{P_{s}-P_{b s}-P_{d s}}{R_{h 1 s}}=\frac{d V_{c s}}{d t_{s}}+\left(\frac{P_{d s}-P_{a s}}{R_{h 2 s}}\right)
$$

Here, $R_{h 1}, R_{h 2}$ and $R_{c}$ are hydraulic resistors. The quantities with a subscript, $s$, are nondimensional values. Values of pressure, volume and time, are non-dimensionalised by corresponding characteristic values $\mathrm{P}_{0}, \mathrm{~V}_{0}$ and $\mathrm{t}_{0}$, respectively. The hydraulic resistors are rescaled by the quantity $\mathrm{P}_{0} \mathrm{t}_{0} / \mathrm{V}_{0}$.

For a rectangular cross-section microchannel of length $L$, width $w$ and height $h$ where $w>h$, the hydraulic resistance to fluid flow due to viscosity $\mu$, is evaluated as (Bruus 2008):

$$
R_{h}(t)=\frac{12 \mu L}{1-0.63(h / w)} \frac{1}{h^{3} w}\left(1-\frac{V(t-d t)-V 0}{L w h}\right)
$$

The quantity in parenthesis accounts for the changing hydraulic resistance as the interface moves along the microchannel with $\mathrm{L}$ as the initial length of microchannel in contact with fluid. The resistance due to gas is neglected as the viscosity of air is $1 \%$ of the water. The above equation is valid for $R_{h 1}$. To calculate the resistance $R_{h 2}$, the parenthesis is replaced with unity. For circular cross section with radius, a, and keeping other quantities same as earlier the hydraulic resistance is obtained from (Bruus 2008):

$$
R_{h}=\frac{8 \mu L}{a^{4} \pi}
$$

Since the value of $R_{c}$ is two orders of magnitude lower than $R_{h 1}$, the resistance $R_{c}$ will be neglected and the pressure $P_{c}$ is taken to be approximately equal to $P_{d}$. It should be noted that the capillary pressure $P_{c}$ is a function of time since the interface will change curvature as liquid bulges out of the stop valve (see Fig 3). The rate of accumulation of fluid at the meniscus by electrical analogy can be formulated as:

$$
\begin{array}{ll}
C_{h} \frac{d P_{c}}{d t} & =\frac{d V_{c}}{d t}=\frac{d V}{d t}-\frac{d V_{R}}{d t} \\
C_{h s} \frac{d P_{c s}}{d t_{s}} & =\frac{P_{s}-P_{b s}-P_{c s}}{R_{h 1 s}}-\frac{P_{c s}-P_{a s}}{R_{h 2 s}}
\end{array}
$$

where $C_{h}=d_{V} / d_{c}$ is hydraulic capacitance of the capillary surface and $C_{h s}$ is the same quantity normalized by $\left(\mathrm{V}_{0} / \mathrm{P}_{0}\right)$. The next challenge is to calculate $\mathrm{C}_{\mathrm{hs}}$ as a function of $\mathrm{P}_{\mathrm{cs}}$.

To do so, we begin with the definition of volume of a spherical cap (Weisstein 2018) shown in Fig. 3a with base radius, a, angle between tangent at the bottom of the cap and the base plane, $\theta$, and $\mathrm{R}=\mathrm{a} / \sin \theta$, as radius of curvature of the spherical surface:

$$
V_{c} \quad=\frac{1}{3} \pi R^{3}\left(2-3 \cos \theta+\cos ^{3} \theta\right)
$$


The value of $\theta$ is time variant and increases as capillary pressure rises. When the value of $\theta$ equals to the contact angle, $\phi$, of the triple line at the edge of the valve, i.e. $\theta=\phi$, capillary barrier is breached and the valve bursts. We will call the volume of fluid accumulated at this point by the capillary surface as threshold volume level and the associated pressure as threshold pressure. This condition is depicted in Fig. 3b.

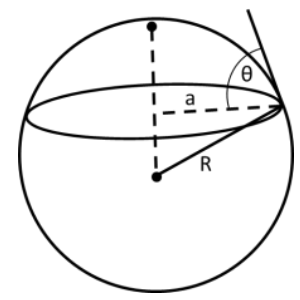

(a)

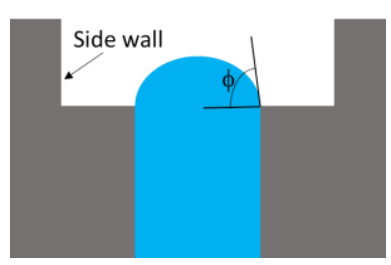

(b)

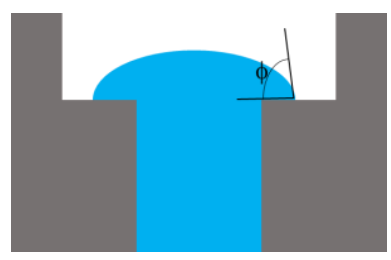

(c)

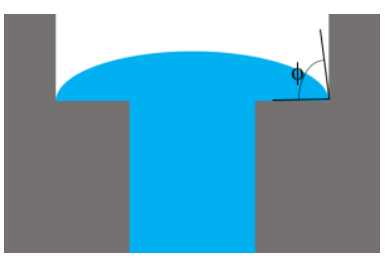

(d)

Fig. 3 (a) Geometry of a spherical cap, (b) cross-section of capillary stop valve showing the meniscus at the burst pressure when $\theta=\phi$, (c) cross-section showing the advancing meniscus after breaching the capillary barrier and (d) cross-section at the moment of valve actuation (when the meniscus makes contact with side walls).

The corresponding pressure for such spherical surface with surface tension $\gamma$ is given by:

$$
P_{c}=\frac{2 \gamma}{R}=\frac{2 \gamma \sin \theta}{a}
$$

If we normalize $\mathrm{V}_{\mathrm{c}}$ and $\mathrm{P}_{\mathrm{c}}$ with $\mathrm{V}_{\mathrm{m}}=(2 / 3) \pi \mathrm{a}^{3}$ and $\mathrm{P}_{\mathrm{m}}=2 \gamma / \mathrm{a}$, respectively, we have from (7)

$$
P_{c}^{\prime}=\frac{P_{c}}{P_{m}}=\sin \theta
$$

and from (6) and (8)

$$
V_{c}^{\prime} \quad=\frac{V_{c}}{V_{m}}=\frac{1}{2 P_{c}^{\prime 3}}\left(2-3\left(1-P_{c}^{\prime 2}\right)^{1 / 2}+\left(1-P_{c}^{\prime 2}\right)^{3 / 2}\right)
$$

Using binomial expansion we get

$$
\begin{aligned}
V_{c}^{\prime} & \approx \frac{1}{2 P_{c}^{\prime 3}}\left(2-3\left(1-\frac{1}{2} P_{c}^{\prime 2}-\frac{1}{8} P_{c}^{\prime 4}-\frac{1}{16} P_{c}^{\prime 6} \ldots\right)+\left(1-\frac{3}{2} P_{c}^{\prime 2}+\frac{3}{8} P_{c}^{\prime 4}+\frac{1}{16} P_{c}^{\prime 6} \ldots\right)\right) \\
& =\frac{1}{8}\left(3 P_{c}^{\prime}+P_{c}^{\prime 3}\right) \\
\frac{d V_{c}^{\prime}}{d P_{c}^{\prime}} & =\frac{3}{8}+\frac{3}{8} P_{c}^{\prime 2} \\
C_{h s} & =\frac{d V_{s}}{d P_{s}}=\frac{d V_{c}}{d P_{c}} \frac{P_{0}}{V_{0}}=\frac{d V_{c}^{\prime}}{d P_{c}^{\prime}} \frac{V_{\mathrm{m}}}{P_{\mathrm{m}}} \frac{P_{0}}{V_{0}} \\
& =\left(\frac{3}{8}+\frac{3}{8} P_{c}^{\prime 2}\right) \frac{V_{\mathrm{m}}}{P_{\mathrm{m}}} \frac{P_{0}}{V_{0}}
\end{aligned}
$$

The burst pressure unhinges the pinned surface at the edge of the stop valve (Fig. 3b) and capillary surface maintains a constant contact angle with the surface (Fig. 3c) till it touches the walls of the tapered microstructure surrounding the valve as shown in Fig. 3d. The volume accumulated by the capillary surface for this situation is defined in our study as actuation volume level and the corresponding capillary pressure as actuation pressure. 
To account for this behavior, we must revise the capacitance formula in equation (12). Since the radius of the base of spherical cap is changing, the non-dimensionalised capillary pressure is

$$
P_{c}^{\prime}=\frac{P_{c}}{P_{m}}=\frac{a}{R}
$$

and the revised non-dimensionalised volume from (5) and (9) is

$$
V_{c}^{\prime} \quad=\frac{1}{2 P_{c}^{\prime 3}}\left(2-3 \cos \theta+\cos ^{3} \theta\right)
$$

which yields the new hydraulic capacitance:

$$
\begin{aligned}
\frac{d V_{c}^{\prime}}{d P_{c}^{\prime}} & =\frac{-3}{2 P_{c}^{\prime 4}}\left(2-3 \cos \theta+\cos ^{3} \theta\right) \\
C_{h s} & =\frac{d V_{s}}{d P_{s}}=\frac{d V_{c}}{d P_{c}} \frac{P_{0}}{V_{0}}=\frac{d V_{c}^{\prime}}{d P_{c}^{\prime}} \frac{V_{\mathrm{m}}}{P_{\mathrm{m}}} \frac{P_{0}}{V_{0}} \\
& =\frac{-3}{2 P_{c}^{\prime 4}}\left(2-3 \cos \theta+\cos ^{3} \theta\right) \frac{V_{\mathrm{m}}}{P_{\mathrm{m}}} \frac{P_{0}}{V_{0}}
\end{aligned}
$$

To solve equation (5), the value of $\mathrm{C}_{\mathrm{hs}}$ is divided into two domains. Before bursting of the valve, $\mathrm{C}_{\mathrm{hs}}$ is given by equation (12) and after the burst it is given by equation (16).

\subsection{Thermal considerations}

In this sub-section, we relate $\mathrm{dV}_{\mathrm{s}} / \mathrm{dt}_{\mathrm{s}}$ in equation (2) to the corresponding rate of rise in gas pressure, $\mathrm{dP}_{\mathrm{s}} / \mathrm{dt}_{\mathrm{s}}$. A beautiful analysis of pressure temperature characteristics of a two-phase water-air system consisting of an enclosed volume is presented in van der Wijngaart et al. 2007. The gas pressure inside the closed volume, by Dalton's Law of Partial Pressures, can be expressed as

$$
P=P_{a}+P_{v}
$$

where $\mathrm{P}_{\mathrm{a}}$ and $\mathrm{P}_{\mathrm{v}}$ are the partial pressures in the trapped air bubble due to dry air and water vapor, respectively. The saturated vapor pressure, $\mathrm{P}_{\mathrm{v}}$, can be estimated using vapor pressure curves like the four-constant equation (Moran and Shapiro 2006, p506):

$$
\begin{aligned}
\ln \left(P_{v}(t)\right) & =A+\frac{B}{T(t)}+C \ln T(t)+D T(t) \\
P_{v}(t) & =\exp \left(A+\frac{B}{T(t)}+D T(t)\right) T(t)^{-C}
\end{aligned}
$$

where, $\mathrm{A}=77.3450, \mathrm{~B}=-7235, \mathrm{C}=8.2, \mathrm{D}=0.0057$, (Engineering Toolbox) and $\mathrm{T}(\mathrm{t})$ is time dependent average gas temperature. After priming, the chamber can be assumed to be at saturated vapor pressure, $\mathrm{P}_{\mathrm{v}}(0)=2400 \mathrm{~Pa}$, at room temperature $\mathrm{T}(0)=293.15 \mathrm{~K}$. 
The pressure temperature relation for the air-water vapor mixture (with mass of air as $\mathrm{m}_{\mathrm{a}}=\frac{\left(\mathrm{P}_{0}-\mathrm{P}_{\mathrm{v}}\right) \mathrm{V}_{0}}{\mathrm{RT}_{0}} \mathrm{M}_{\mathrm{a}}$, where, $\mathrm{M}_{\mathrm{a}}$ is molar mass of air, can be explained by the ideal gas equation:

$$
P V=P_{a} V+P_{v} V=m_{a} R_{a} T+P_{v} V
$$

Taking derivative on both sides we have

$$
\begin{aligned}
P \frac{d V}{d t}+V \frac{d P}{d t} & =m_{a} R_{a} \frac{d T}{d t}+\frac{d}{d t} P_{v} V \\
\frac{P_{0} V_{0}}{t_{0}}\left(P_{a s} \frac{d V_{s}}{d t_{s}}+V_{s} \frac{d P_{s}}{d t_{s}}\right) & =\rho_{0} V_{0} \rho_{s} V_{s} R_{a} \frac{T_{0}}{t_{0}} \frac{d T_{s}}{d t_{s}}+\left(V_{s} \frac{d P_{v s}}{d t_{s}}+P_{v s} \frac{d V_{s}}{d t_{s}}\right) \frac{P_{0} V_{0}}{t_{0}} \\
\frac{d P_{s}}{d t_{s}} & =\frac{1}{V_{s}}\left(R_{s} \frac{d T_{s}}{d t_{s}}-P_{s} \frac{d V_{s}}{d t_{s}}\right)+\frac{1}{V_{s}}\left(V_{s} \frac{d P_{v s}}{d t_{s}}+P_{v s} \frac{d V_{s}}{d t_{s}}\right)
\end{aligned}
$$

$\mathrm{R}_{\mathrm{as}}=\mathrm{R}_{\mathrm{a}} /\left(\mathrm{P}_{0} / \rho_{0} \mathrm{~T}_{0}\right)$ is gas constant for air, where, $\rho_{0}$ and $\mathrm{T}_{0}$ as characteristic values of gas density and temperature respectively. The rate of vapor pressure rise in the bubble, $\mathrm{dP}_{\mathrm{vs}} / \mathrm{dt}_{\mathrm{s}}$, is computed as follows:

$$
\frac{d P_{v s}(t)}{d t_{s}}=\frac{P_{v}(t+d t)-P_{v}(t)}{d t} \frac{t_{0}}{P_{0}}
$$

The expression for $\mathrm{dP}_{\mathrm{s}} / \mathrm{dt}_{\mathrm{s}}$ in equation (20) always corresponds to saturated condition. This might not be true in actual process and may lead to overestimation of the pressure rise. We insert a factor $\mathrm{k}_{\mathrm{v}}$ such that $0<\mathrm{k}_{\mathrm{v}}<=1$, to control the degree of vapor fraction in the heated bubble as shown in the equation (22). Since there is always some moisture in the bubble, $\mathrm{k}_{\mathrm{v}}$ is always greater than 0 and $\mathrm{k}_{\mathrm{v}}=1$ corresponds to saturated condition.

$$
\frac{d P_{s}}{d t_{s}}=\frac{1}{V_{s}}\left(R_{s} \frac{d T_{s}}{d t_{s}}-P_{s} \frac{d V_{s}}{d t_{s}}\right)+\frac{k_{v}}{V_{s}}\left(V_{s} \frac{d P_{v s}}{d t_{s}}+P_{v s} \frac{d V_{s}}{d t_{s}}\right)
$$

In equation (18) and (22), $\mathrm{T}_{\mathrm{s}}$ and $\mathrm{dT}_{\mathrm{s}} / \mathrm{dt}_{\mathrm{s}}$, respectively, are the unknown input quantities. We perform a fully coupled finite element simulation on a 3D model of the device to extract these data in the next sub-section.

\subsection{Finite Element Analysis (FEA)}

A fully coupled electrothermal FEA simulation is conducted using COMSOL 5.2a (to determine $\mathrm{T}_{\mathrm{s}}$ and $\mathrm{dT}_{\mathrm{s}} / \mathrm{dt}_{\mathrm{s}}$ ) on the geometry as shown in Fig.4. The trapped gas is indicated in blue which is surrounded, by silicon, water and glass. The silicon block on top of the trapped gas has an aluminum microheater and is surrounded by air domain which acts as thermal insulation. For the purposes of the FEA simulation, the volume of the trapped gas is assumed to be constant. This significantly reduces the computational complexity of the problem since the liquid-gas interface can be treated as a static surface (i.e., neglecting the two-phase fluid transport and movement of the interface). Additionally, evaporation is neglected in the FEA model and dry air properties are applied to the air domain. 


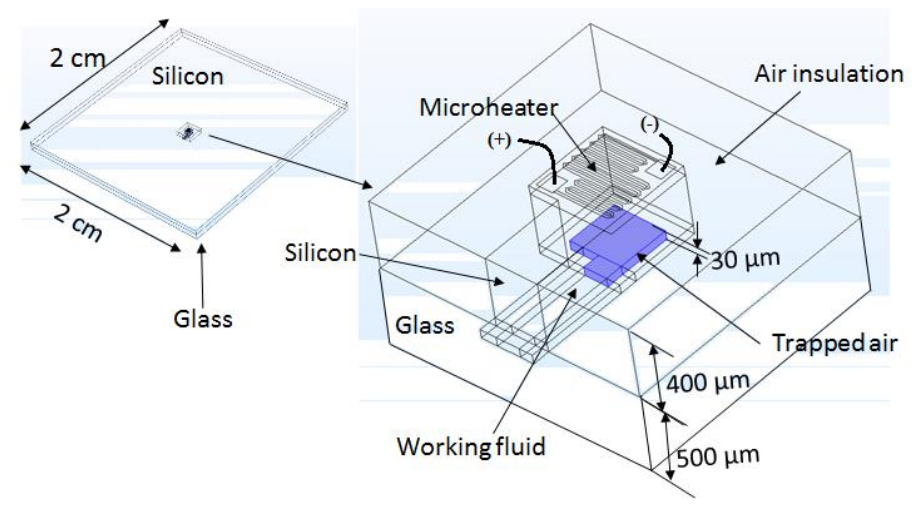

Fig. 4 Computational domain and dimensions for FEA model.

The work done by the expanding gas is neglected since it is insignificant. Heat conduction within the computational domain is computed with the heat diffusion equation (Lienhard and Lienhard 2015):

$$
\rho C_{p} \frac{\partial T}{\partial t}+\vec{\nabla} \cdot \vec{q}=Q_{v}
$$

where, $Q_{v}, \rho$ and $C_{p}$ are volumetric the heat source generation rate, mass density, and specific heat, respectively. By Fourier's law of heat conduction, $\vec{q}=-k \overrightarrow{\nabla T}$ is the heat flux with $k$ being the thermal conductivity. Heat generated in aluminum heater is calculated as $Q_{v}=\vec{J} \cdot \vec{J} \rho_{r}$ with $\overrightarrow{\mathbf{J}}$ as the electrical current density and $\rho_{\mathrm{r}}$ as the aluminum electrical resistivity. The resistivity is described by $\overrightarrow{\mathrm{J}}=\sigma_{\mathrm{r}} \overrightarrow{\mathrm{E}}$, where, $\sigma_{\mathrm{r}}$ is the electrical conductivity $\left(37.7 \times 10^{6} \mathrm{~S} / \mathrm{m}\right.$ for aluminum), $\overrightarrow{\mathrm{E}}=-\vec{\nabla}_{\mathrm{t}} \mathrm{V}$ is the electrical field intensity and $\mathrm{V}$ is the electrical potential.

The current conservation in the aluminum microheater is accounted for by the following equation:

$$
-\vec{\nabla}_{\mathrm{t}} \cdot \overrightarrow{\mathrm{J}}=0
$$

The aluminum heater is modeled as a high thermally conductive thin layer with $\mathrm{k}_{\mathrm{T}}$ as the thermal conductivity and $\mathrm{d}_{\mathrm{T}}=300 \mathrm{~nm}$ as the thickness using COMSOL's thin layer feature, which neglects the component of the heat flux within the plane of the heater. The heat source is coupled to the domain by a heat flux boundary normal with $\vec{n}$ as outward unit vector normal to heater surface using:

$$
-\overrightarrow{\mathrm{n}} \bullet\left(-\mathrm{k}_{\mathrm{T}} \vec{\nabla} \mathrm{T}\right)=\mathrm{d}_{\mathrm{T}} \rho_{\mathrm{s}} \mathrm{C}_{\mathrm{p}, \mathrm{s}} \frac{\partial \mathrm{T}}{\partial \mathrm{t}}-\vec{\nabla}_{\mathrm{t}} \cdot\left(\mathrm{d}_{\mathrm{T}} \mathrm{k}_{\mathrm{T}} \vec{\nabla}_{\mathrm{t}} \mathrm{T}\right)
$$

Where, $C_{p, s}$ and $\rho_{s}$ are the specific heat and density of the aluminum layer, respectively. Subscript, $t$, in the last term indicates the tangential gradient.

The heat interaction of the domain through convection is accounted by giving a nominal convective heat transfer coefficient $\mathrm{h}=2 \mathrm{~W} / \mathrm{K} / \mathrm{m}^{2}$. Radiative losses are neglected since the operating temperature of the device is relatively low. 


\subsection{Analytical Model computation}

After extracting the temperature $T_{\mathrm{s}}$ and temperature derivative $\mathrm{dT}_{\mathrm{s}} / \mathrm{dt}_{\mathrm{s}}$ with respect to time from FEA model we can now solve the equations (2), (5) and (19) simultaneously with following initial conditions:

$$
V_{S}(0)=1 ; \quad P_{S}(0)=1 ; \quad P_{C S}(0)=\frac{P_{a}}{P_{0}} ; \quad \rho_{0}=\frac{m_{a}}{V_{0}}
$$

This initial value problem is implemented using the ordinary differential equation solver ODEINT, available in the SciPy package of Python 3.6, an opensource high level scripting language. This exercise gives the variation of gas volume, $\mathrm{V}_{\mathrm{s}}$, and pressure, $\mathrm{P}_{\mathrm{s}}$, along with pressure reached at the stop valve, $\mathrm{P}_{\mathrm{cs}}$, with respect to time. The output of this exercise is reported and discussed in the results section.

\section{Fabrication}

The device is fabricated on $200 \mathrm{~mm}$ diameter silicon and Pyrex glass wafers. The fabrication process consists of three silicon deep reactive ion etch steps, one anodic bonding and one metal etching steps. The micro-channels and bubble chamber are fabricated by a shallow, $30 \mu \mathrm{m}$ etch from one side of silicon surface and a deeper, $200 \mu \mathrm{m}$ etch defines liquid reservoirs and the stop valve openings using a process flow similarly reported by Majeed et al. (2015). Next, these structures are sealed by anodic bonding to a Pyrex wafer. The backside of the silicon is ground to a thickness of $400 \mu \mathrm{m}$. A $300 \mathrm{~nm}$ aluminum layer is deposited on the back of PCR chamber and then etched into a serpentine of $20 \mu \mathrm{m}$ width to form the heater. To form access holes and the open microchannel structure, a third etch from the silicon backside, approximately $200 \mu \mathrm{m}$ deep, merges with the frontside deep etch of the reservoirs and stop valve structures.

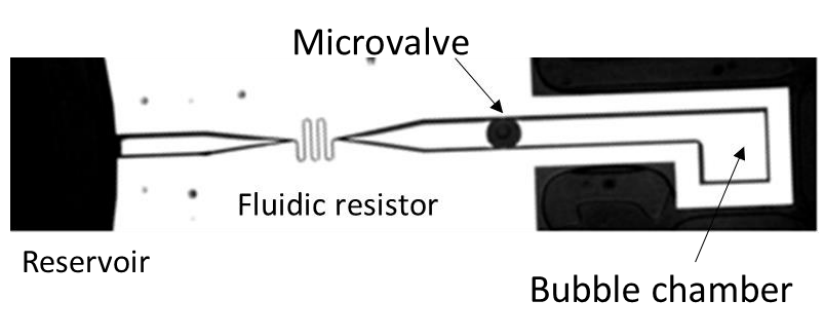

(a)

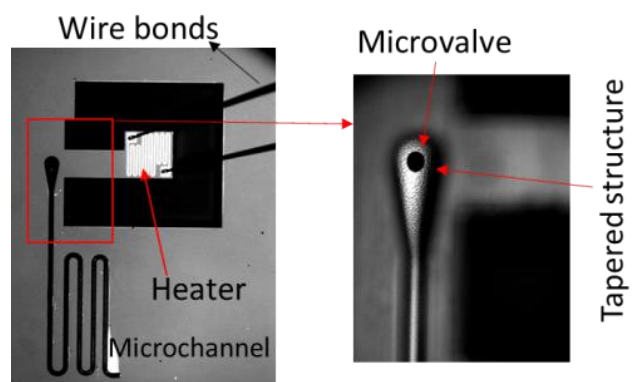

(b)

Fig. 5 (a) Micrograph of fabricated device from seen from the glass side and (b) silicon side view of the device showing microheater, bond wires, microvalve and microchannel.

Fig. 5a shows the glass side view of the fabricated device with various microstructures and Fig. $5 \mathrm{~b}$ shows the silicon side with microheater and microvalve. A taper structure surrounds the microvalve to increase curvature of the liquid-gas interface, and thus facilitate capillary flow.

\section{Experiments}

The silicon test chip (a $2 \mathrm{~cm} \times 2 \mathrm{~cm}$ die) is glued to a PCB with the access holes for the reservoirs and the open microchannels facing the PCB. A cutout in the PCB underneath the silicon chip allows fluidic access. $25 \mu \mathrm{m}$ diameter aluminum wires connect to the bond pads on the microheater from the PCB. The PCB is connected to a Keithley source-meter 2602 via 
a 20-pin connector. The chip/PCB combination as shown in Fig. 6a is placed on an inverted microscope with the glass side facing the microscope objective lens. A high-speed camera is attached to the inverted microscope. To capture the event from the silicon side, an upright microscope with a second high-speed camera is installed over the chip. The cameras are synchronized via. a camera hub and triggered simultaneously by a voltage trigger pulse sent from source-meter prior to applying the voltage signal to the heater on the silicon chip. A PC running Keithley's Test Script Builder (TSB) is used to control and program the source-meter. The schematic of the experiment set up is depicted in Fig. 6 b.

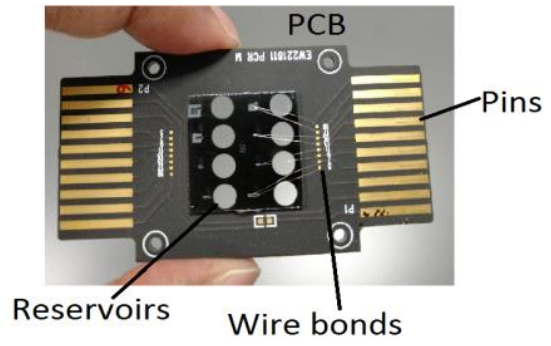

(a)

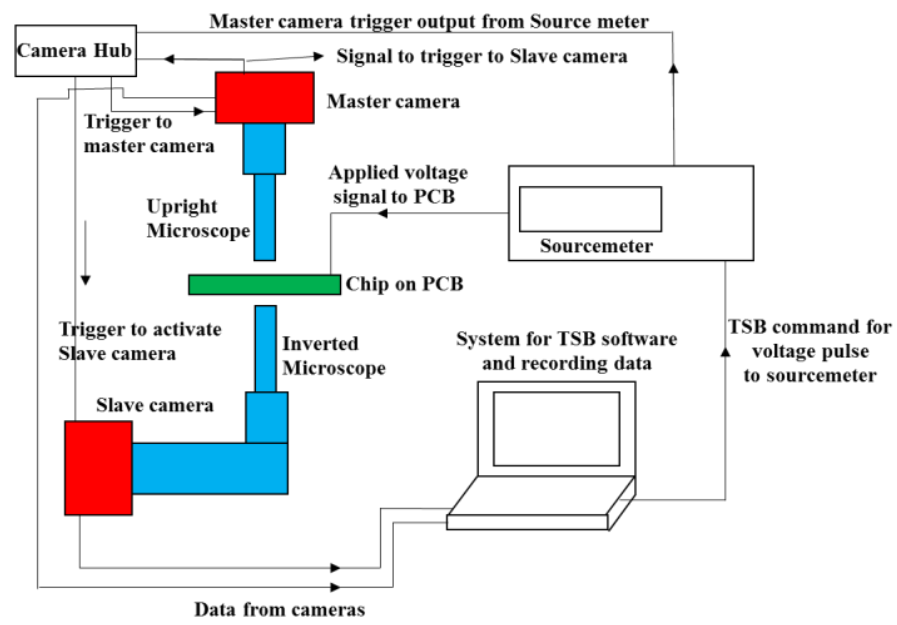

(b)

Fig. 6 (a) Silicon/Pyrex chip mounted on a PCB and (b) experimental set up

De-ionized water as working fluids is employed for the experiments De-ionized water has a surface tension of $72 \mathrm{mN} / \mathrm{m}$ and contact angle of $65^{\circ}$. To render surfaces with uniform contact angle the surfaces were silanized with PEG coating beforehand.

\section{Results}

\subsection{FEA output}

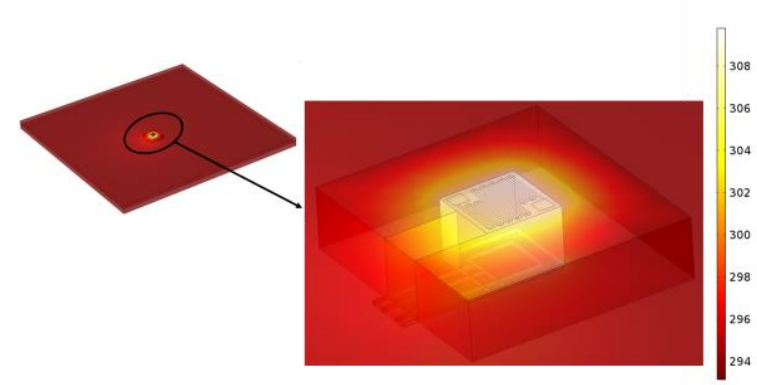

(a)

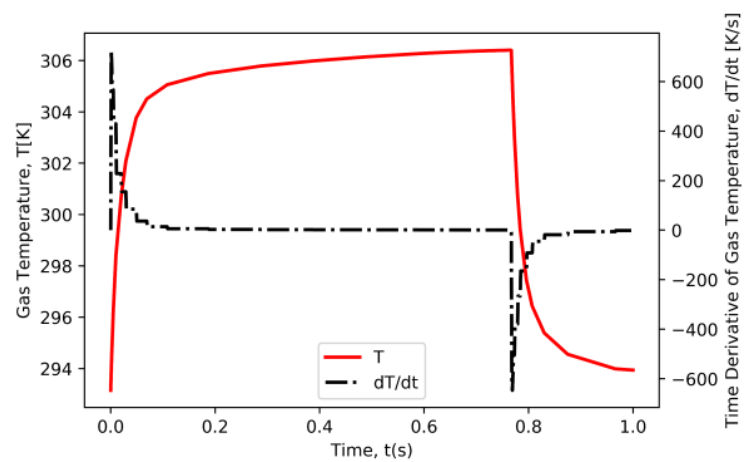

(b)

Fig. 7 (a) Temperature map of chip at $t=0.5 \mathrm{~s}$ and (b) temperature profile of gas bubble, $\mathrm{T}$ and its time derivative, dT/dt.

Figure 7a shows temperature map of the whole chip and enlarged microheater region during a $780 \mathrm{~ms}$ voltage pulse of $3 \mathrm{~V}$ at $\mathrm{t}=0.78 \mathrm{~s}$ when the temperature of the chip peaks. It can be noticed that the there is a sharp temperature gradient around the heater due to the air insulation. The rest of the chip mostly remains at the room temperature due to the combined effect of air insulation, small duration of the pulse and low power input. The values of the temperatures of 
trapped air bubble and its time derivative are depicted in Fig. 7b. It can be noticed that only at the beginning and at the end of the voltage pulse, the rate of temperature change has noticeable values. For the rest of the time, it is a flat curve near the zero value. This gas temperature, $T_{s}$, along with its time derivative, $\mathrm{dT}_{\mathrm{s}} / \mathrm{dt}_{\mathrm{s}}$, extracted from the finite element analysis is the starting point to solve the analytical model in section 3.4.

\subsection{Experimental and Numerical results}

\subsubsection{Temperature measurements}

The electrothermal finite element analysis is validated by comparing predicted heater temperatures to experimental measurements. For this, we consider the average temperature value of the heater domain from the FEA model. Calibration of the joule heater by experimental measurements in an oven determined that the temperature coefficient of resistance (TCR), $\alpha$, is approximately $0.0039 \mathrm{~K}^{-1}$ where $\mathrm{R}_{\mathrm{t}+\mathrm{dt}}=\mathrm{R}_{\mathrm{t}}\left(1+\alpha\left(\mathrm{Th}_{\mathrm{t}+\mathrm{dt}}-\mathrm{Th}_{\mathrm{t}}\right)\right)$ describes the relationship between heater resistance, $\mathrm{R}$, heater temperature, $\mathrm{Th}$, and the TCR value. The subscripts $t$ and $\mathrm{t}+\mathrm{dt}$ refer to the current and the next time steps, respectively. The resistance of the heater is determined using Ohm's law of conduction, $\mathrm{R}=\Delta \mathrm{V} / \mathrm{I}$ where, $\Delta \mathrm{V}$ is the applied voltage difference and I is current. The current is measured using the Keithley source-meter with a time interval of $5 \mathrm{~ms}$.

Figure 8 depicts the comparison between experiment and FEA simulation for $0.75 \mathrm{~s}$ duration electrical potential pulses of $3 \mathrm{~V}$ and $9 \mathrm{~V}$. The simulation results slightly over-estimate the heater temperature during the initial phase of the voltage pulse $(<200 \mathrm{~ms})$. A possible explanation of this over-prediction is that the thermal contact resistance between the microheater and silicon substrate is neglected in the simulation. Thus, the average surface temperature of the microheater might incorporate some thermal lag due to the silicon mass. For the rest of the curve the values of the experiment and simulation are very similar. This means, we can safely feed the temperature values of simulation to the analytical model.

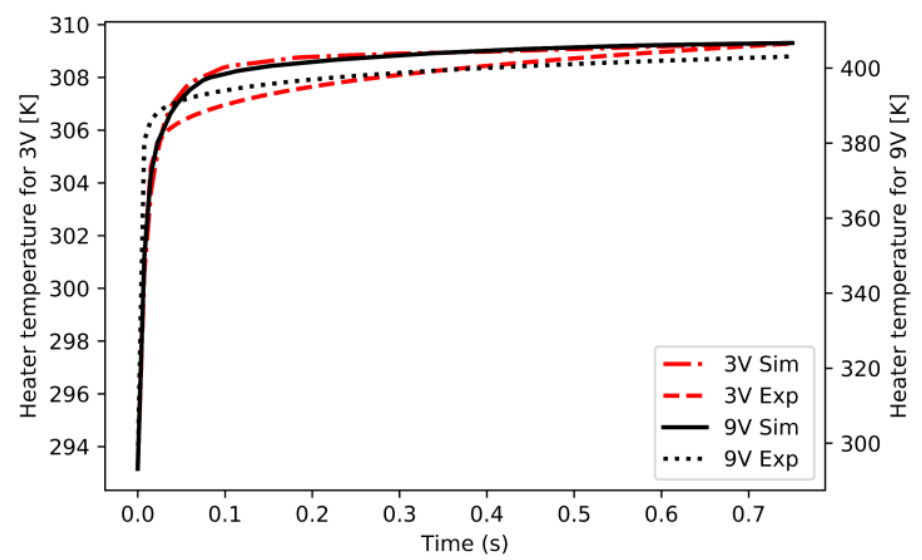

Fig. 8 Comparison between FEA simulation results and experimental measurements of the average heater temperature. 


\subsubsection{Actuation of capillary valve}

De-ionized water with surface tension of $0.072 \mathrm{~N} / \mathrm{m}$ is used as working fluid to prime the device. The capillary flow from reservoir towards the chamber inside the microchannel traps an air bubble of initial gas volume $2.2 \times 10^{-12} \mathrm{~m}^{3}$ as shown in Fig. 9a at time $\mathrm{t}=0 \mathrm{~s}$. The contact angle of the substrate and the working fluid was found to be around $65^{\circ}$ and hydraulic resistance of the fluidic resistor computed to be about $3.6 \times 10^{15} \mathrm{~Pa} \mathrm{~s} / \mathrm{m}^{3}$. We required $5.5-6 \mathrm{~V}$ to burst the valve. The meniscus pressure overcomes the capillary barrier at around $t=15 \mathrm{~ms}$ and then the contact line advances radially as depicted in Fig. $3 \mathrm{~b}$ for $15 \mathrm{~ms}$ towards the vertical walls. Figure $9 \mathrm{~b}$ shows the expansion of the meniscus at $\mathrm{t}=30 \mathrm{~ms}$, when the valve is about to actuate. The contact of the meniscus with the sidewalls actuates the valve at around $35 \mathrm{~ms}$. When the gas cools down, the contraction of the bubble pulls the meniscus back into the vertical hole as can be seen at $t=815 \mathrm{~ms}$, Fig. 12c. This contraction of the gas bubble pulls the fluid inside the microchannel and inhibits the capillary flow in the outer channel. This is a drawback of the current design.

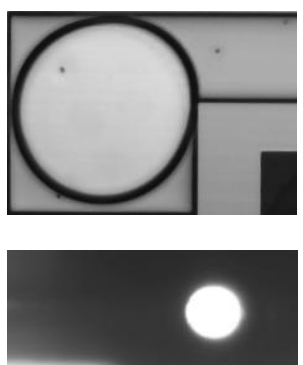

(a)
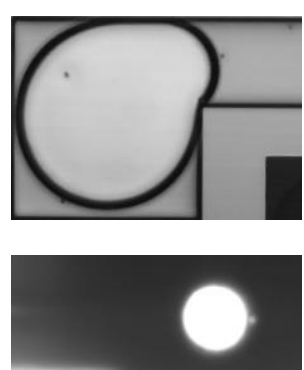

(b)
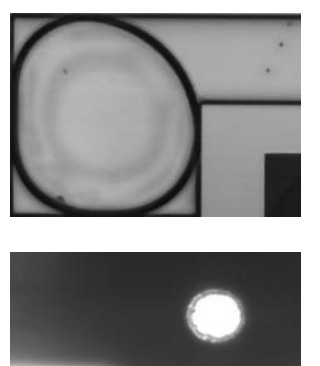

(c)

Fig. 9 Micrographs of bubble at (a) $t=0 \mathrm{~s}$, (b) $t=30 \mathrm{~ms}$ (after capillary barrier breaching and just before actuation) and (c) $\mathrm{t}=815 \mathrm{~ms}$ (after contraction)

\subsubsection{Effect of hydraulic resistance}

The hydraulic resistance is a critical parameter in the performance of the device. For similar energy input, higher resistance reduces the backflow and more fluid is directed towards the valve meniscus which accelerates the bursting and actuation of the valve. A comparison of the backflows for different resistance values confirms this. We used the actual device hydraulic resistance of $3.6 \times 10^{15} \mathrm{~Pa} . \mathrm{s} / \mathrm{m}^{3}$ and another with an order less, $3.6 \times 10^{14} \mathrm{~Pa} . \mathrm{s} / \mathrm{m}^{3}$. The simulation results of these cases are plotted in Figs. 10(a) and 10(b) respectively. It is clearly evident, that the backflow increases significantly with an order lower hydraulic resistance. The volume of fluid accumulated by the capillary surface, depicted in the Fig. 10(a) as meniscus volume, surpasses the threshold volume level or the burst volume level at around $t=9 \mathrm{~ms}$ for the higher hydraulic resistance with almost negligible backflow and therefore, the gas volume expansion is equivalent to meniscus volume in this case. 


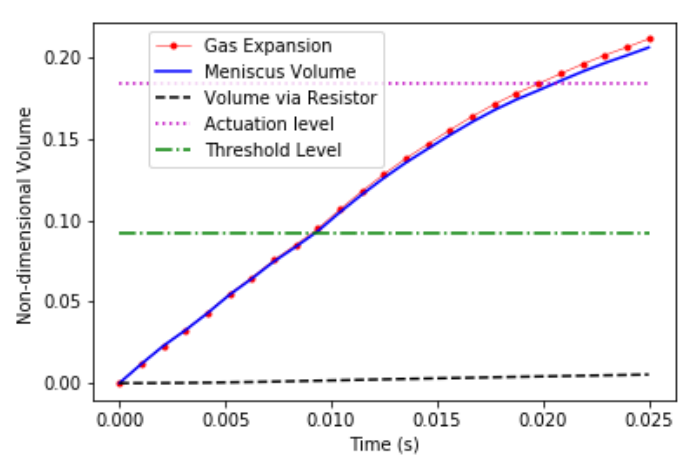

(a)

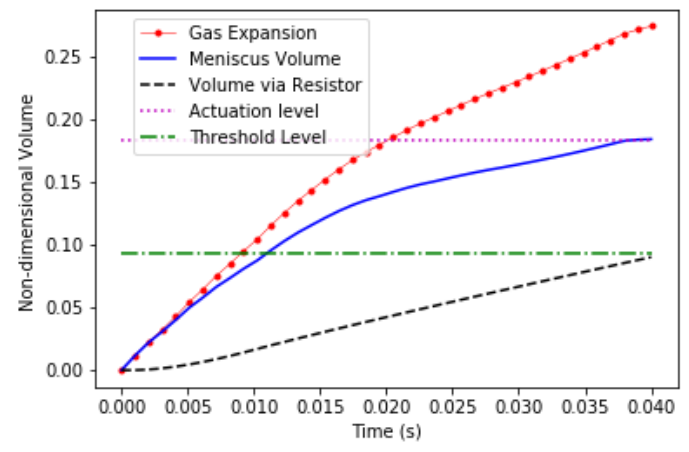

(b)

Fig. 10 Gas expansion curves for saturated condition, $k_{v}=1$, for (a) $R_{h 2}=3.6 \times 10^{15} \mathrm{~Pa} . \mathrm{s} / \mathrm{m}^{3}$ and (b) $R_{h 2}=3.6 \times 10^{14} \mathrm{~Pa} . \mathrm{s} / \mathrm{m}^{3}$

From Fig. 10(b), the threshold volume is breached 2 ms later for the lower hydraulic resistance. The gas expansion as can be seen from Fig. 10b, coincides with the meniscus volume only for about $5 \mathrm{~ms}$, after which it diverges as gas pressure increases and consequently the backflow through the resistor also rises. There is a significant delay in the actuation of the valve for the later case because of high backflow. The meniscus volume breaches the actuation volume at around $40 \mathrm{~ms}$, about $18 \mathrm{~ms}$ later than the former case which gets actuated at around $22 \mathrm{~ms}$.

\subsubsection{Effect of $\mathrm{k}_{\mathrm{v}}$}

The exact thermodynamic process during the gas expansion is unknown but we can have a fair idea about the degree of saturation of gas bubble by comparing gas expansion simulation curves for different value of $\mathrm{k}_{\mathrm{v}}$ with the experimental result. The time rate change of gas bubble volume is determined via image processing with a custom-made Matlab code. Fig. 11a shows processed images at $\mathrm{t}=0 \mathrm{~ms}$ (top) and $300 \mathrm{~ms}$ (bottom), respectively. The images are converted into binary and the summation of pixel values is determined with a pixel value of 1 (white pixels) indicating the gas phase and 0 indicating the liquid phase. The volume of each pixel is taken as the area per pixel (calculated using MATLAB based on image resolution) and the depth of the microchannel $(30 \mu \mathrm{m})$.

We simulate saturated and dry air conditions corresponding to $\mathrm{k}_{\mathrm{v}}=1$ and $\mathrm{k}_{\mathrm{v}}=0$, respectively. Both these conditions are idealistic and found to be unsatisfactory to represent the reality as can be seen from the experimental measurement (Exp) and analytical model predictions of the volume ratio of gas bubble, $\mathrm{V}(\mathrm{t}) / \mathrm{V}_{0}$, as shown in Fig. 11a. The saturated curve bursts and actuates the valve much earlier than the experimental curve, roughly around at $\mathrm{t}=8 \mathrm{~ms}$ and at $\mathrm{t}=18 \mathrm{~ms}$ respectively, whereas, the experimental curve suggests the valve to have reached threshold level at around $15 \mathrm{~ms}$ and the actuation volume level at around $33 \mathrm{~ms}$. The dry air condition curve only manages to breach the capillary barrier at around $t=36 \mathrm{~ms}$, much later than the experimental curve but for the same energy input, the gas does not even expand enough to push the capillary surface up to the sidewalls and actuate the valve.

Next, we adopt a hit and trial method for various values of $\mathrm{k}_{\mathrm{v}}$, so that the analytical model approaches the actual process. We found that $\mathrm{k}_{\mathrm{v}}=0.6$ is good estimate. The actuation time of the valve is very similar with that of the experiment. The approximation can be further improved by adopting a time variant value of $\mathrm{k}_{\mathrm{v}}$ instead of a constant value, since the degree of evaporation depends on temperature which is continuously changing with time during the process. In Fig. 11a, the experimental curve is first concave upwards and later turns convex whereas, all the analytical model curves are convex upwards from the beginning. This can be explained by the reduced rise of temperature in the experiment due to latent heat of evaporation. 
The model incorporates the vapor pressure at the temperature provided by the FEA but does not include the endothermic effect of evaporation.

The pressure characteristics of the analytical model for $\mathrm{k}_{\mathrm{v}}=0.6$ is shown in Fig. $11 \mathrm{~b}$. The gas pressure can be seen to peak above $2.2 \%$ of $\mathrm{P}_{0}$ corresponding to the point when the valve pressure touches burst pressure. After the burst, the valve pressure curve traces a negative slope given due to negative hydraulic capacitance given by equation (10) and finally it drops to the actuation pressure level of the valve at around $35 \mathrm{~ms}$.

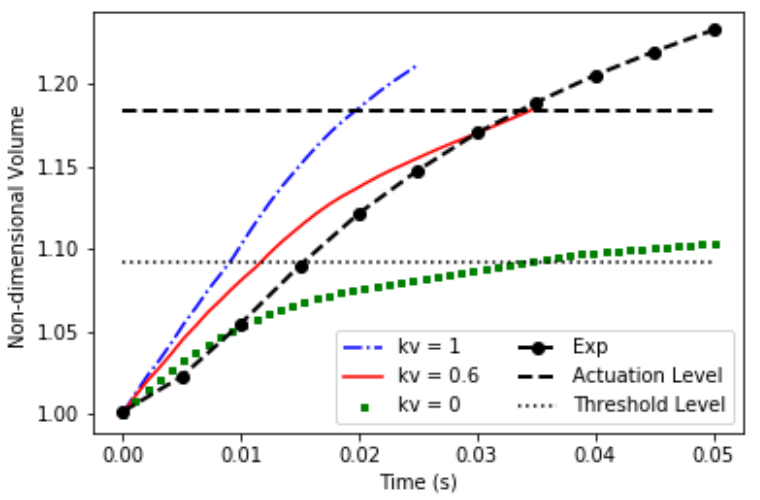

(a)

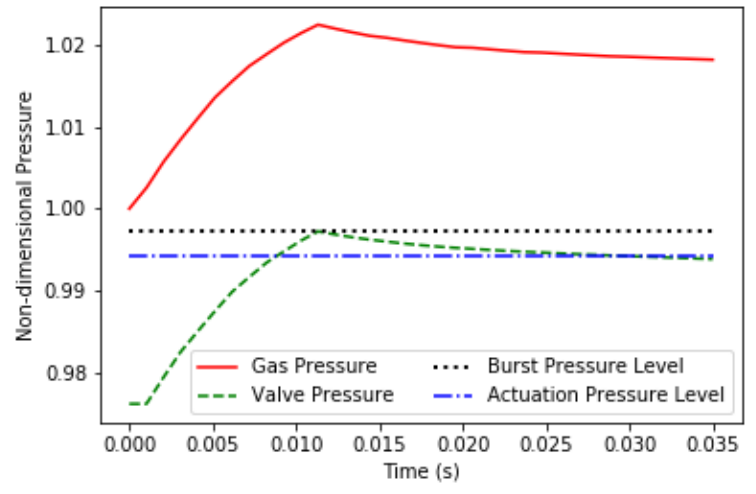

(b)

Fig. 11 (a) Experimental and simulation results of volume expansion (b) Pressure profile for gas and valve meniscus for $\mathrm{k}_{\mathrm{v}}$ $=0.6$.

\section{Conclusion}

We demonstrated a compact low voltage actuation of thermo-pneumatic actuation of capillary stop valve in silicon and glass wafers. The design is done keeping in mind the existing flow process for micro-PCR chip in our facility. An analytical model is constructed along with finite element simulations to understand the system. The model gives good insight on the performance characteristics of the system. Even if the model does not give exact results, it estimates the influence of evaporation on the system behavior. Use of high-speed infrared camera can accurately measure the evaporation and would provide far better consistency between experimental and analytical results. Image analysis of the expanding bubble accurately quantifies the extend of expansion.

\section{Acknowledgements}

This work is funded by the Life Science Technologies, IMEC, Heverlee, Belgium. The authors also thank Frank Gijbels of IMEC for extending help for designing the PCB.

\section{References}

Temiz Y, Lovchik RD, Kaigala GV, Delamarche E (2015) Lab-on-a-chip devices: How to close and plug the lab? Microelectronic Engineering 132:156-175. http://dx.doi.org/10.1016/j.mee.2014.10.013

Oh KW, Chong HA (2006)TOPICAL REVIEW: A review of microvalves J. Micromech. Microeng,16, Issue 5: R13R39 
Grover WH, Skelley AM, Liu CN, Lagally ET, Mathies RA (2003) Monolithic membrane valves and diaphragm pumps for practical large-scale integration into glass microfluidic devices, Sensors and Actuators B: Chemical, 89(3): 315-323 https://doi.org/10.1016/S0925-4005(02)00468-9.

Kazuo Hosokawa and Ryutaro Maeda (2000) A pneumatically-actuated three-way microvalve fabricated with polydimethylsiloxane using the membrane transfer technique, J. Micromech. Microeng. 10 415, https://doi.org/10.1088/0960-1317/10/3/317

Melin J and Quake SR (2007) Microfluidic Large-Scale Integration: The Evolution of Design Rules for Biological Automation Annual Review of Biophysics and Biomolecular Structure 2007 36:1, 213-231. https://doi.org/10.1146/annurev.biophys.36.040306.132646

Shaegh SAM, Wang Z, Ng SH, Chan LCZ, Toh AGG, Wang Z (2015) Plug-and-play microvalve and micropump for rapid integration with microfluidic chips. Microfluid Nanofluid 19: 557. https://doi.org/10.1007/s10404-015-1582-4

Pilarski PM, Adamia S, Backhouse CJ (2005) An adaptable microvalving system for on-chip polymerase chain reactions. Journal of Immunological Methods 305: 48 - 58 doi:10.1016/j.jim.2005.07.009

Juncker D, Schmid H, Drechsler U, Wolf H, Wolf M, Michel B, de Rooij N, Delamarche E (2002) Autonomous Microfluidic Capillary System Anal. Chem. 74,24, 6139-6144 https://doi.org/10.1021/ac0261449

Irimia D. (2008) Capillary Force Valves. In: Li D. (eds) Encyclopedia of Microfluidics and Nanofluidics. Springer, Boston, MA

Man PF, Mastrangelo CH, Burns MA and Burke DT, "Microfabricated capillarity-driven stop valve and sample injector," Proceedings MEMS 98. IEEE. Eleventh Annual International Workshop on Micro Electro Mechanical Systems. An Investigation of Micro Structures, Sensors, Actuators, Machines and Systems (Cat. No.98CH36176, Heidelberg, Germany, 1998, pp. 45-50. doi: 10.1109/MEMSYS.1998.659727

Eriksen J, Bilenberg B, Kristensen A, and Marie R (2017) Optothermally actuated capillary burst valve, Review of Scientific Instruments 88, 045101 (2017); https://doi.org/10.1063/1.4979164

Cho H, Kim HY, Kang JY, Kim TS (2007) How the capillary burst microvalve works, Journal of Colloid and Interface Science, Volume 306(2), 379-385, https://doi.org/10.1016/j.jcis.2006.10.077.

Barman U, Wiederkehr RS, Fiorini P, Lagae L, Jones B (2018) A comprehensive methodology for design and development of an integrated microheater for on-chip DNA amplification. J. Micromech. Microeng, 28, 085014, doi:10.1088/1361-6439/aabd2c

Abi-Samra, K., Clime, L., Kong, L. et al. Microfluid Nanofluid (2011) 11: 643. https://doi.org/10.1007/s10404-011$\underline{0830-5}$

Handique K, Burke DT, Mastrangelo CH, Burns MA (2000) Nanoliter Liquid Metering in Microchannels Using Hydrophobic Patterns Anal. Chem. 72: 4100-4109 DOI: 10.1021/ac000064s

van der Wijngaart W, Chugh D, Man E, Melin J, Stemme J, (2007) A Low-Temperature Thermopneumatic Actuation Principle for Gas Bubble Microvalves. Journal of Microelectromechanical Systems, 16 (3),765-774. doi: 10.1109/JMEMS.2007.893514

Bohm S, (2006) Triggerable Passive Valve for use in Controlling the Flow of Fluid US 7,059,352 B2

Oh KW, Lee K, Ahn B, Furlani EP (2012) Design of pressure-driven microfluidic networks using electric circuit analogy. Lab Chip. 12(3):515-45. doi: 10.1039/c2lc20799k

Bruus H, (2008) Theoretical Microfluidics, $1^{\text {st }}$ Ed. Oxford University Press pp 75

Weisstein, EW. "Spherical Cap." From MathWorld--A Wolfram Web Resource. http://mathworld.wolfram.com/SphericalCap.html accessed on Dec 102018

Moran MJ, Shapiro HN, (2006) Fundamentals of Engineering Thermodynamics $5^{\text {th }}$. Ed. Wiley, West Sussex, pp 506

\section{https://www.engineeringtoolbox.com/}

Ranjan R, Patel A, Garimella SV, Murthy JY (2012), Wicking and thermal characteristics of micropillared structures for use in passive heat spreaders, International Journal of Heat and Mass Transfer, 55(4) : 586-596, https://doi.org/10.1016/j.ijheatmasstransfer.2011.10.053. 
B. Majeed, A. Taher, B. Jones and D. Sbuncuoglu, "Two step silicon microfluidics for capillary valve applications," International Symposium on Microelectronics, 2015, 555 (2015).

Lienhard IV, Lienhard V, (2015) A Heat Transfer Textbook, Phlogiston Press, Cambridge, pp. 55

https://www.sigmaaldrich.com 